\title{
Quantifying the relationship between cell division angle and morphogenesis through computational modeling
}

\author{
Emma Lejeune, Christian Linder* \\ Department of Civil and Environmental Engineering, Stanford University, Stanford, California, 94305, USA
}

\begin{abstract}
When biological cells divide, they divide on a given angle. It has been shown experimentally that the orientation of cell division angle for a single cell can be described by a probability density function. However, the way in which the probability density function underlying cell division orientation influences population or tissue scale morphogenesis is unknown. Here we show that a computational approach, with thousands of stochastic simulations modeling growth and division of a population of cells, can be used to investigate this unknown. In this paper we examine two potential forms of the probability density function: a wrapped normal distribution and a binomial distribution. Our results demonstrate that for the wrapped normal distribution the standard deviation of the division angle, potentially interpreted as biological noise, controls the degree of tissue scale anisotropy. For the binomial distribution, we demonstrate a mechanism by which direction and degree of tissue scale anisotropy can be tuned via the probability of each division angle. We anticipate that the method presented in this paper and the results of these simulations will be a starting point for further investigation of this topic.
\end{abstract}

Keywords: cell division, morphogenesis, peridynamics

2010 MSC: 74L15, 92C10

*Corresponding Author. Email address: linder@stanford.edu

Preprint submitted to Journal of Theoretical Biology

November 23, 2016

(C) 2017. This manuscript version is made available under the Elsevier user license

http://www.elsevier.com/open-access/userlicense/1.0/ 


\section{Introduction}

Significant effort has been made toward developing rules for predicting division angle in an individual cell (Gillies and Cabernard, 2011). For example, external cues such as peptide gradients (Lamb et al. 2014), applied stretch (Wyatt et al., 2015), and applied force (Nestor-Bergmann et al. 2014) have all been shown to influence division angle. Geometric factors such as cell geometry (Su et al. 2015$)$ and cell packing geometry (Gibson et al. 2011) also play a role. In studies of cell division orientation, it is typical to report a histogram of different experimentally observed division angles corresponding to a fixed experimental set up, similar to the histogram shown in Fig. 17. Based on this experimentally observed variability and the fact that the subcellular mechanism driving cell division are noisy (Akanuma et al. 2016 Bosveld et al., 2016; Corrigan et al., 2015, Juschke et al., 2013), it follows that cell division angle is best understood as a random variable. However, it is still unknown how the stochastic cell division angle influences morphogenesis on the population and tissue scales (Matamoro-Vidal et al., 2015; Minc and Piel 2012). Here we take a simulation based initial approach to answering this fundamental question: how does the underlying distribution of division orientations influence morphogenesis?

To better understand how division angle orientation influences morphogenesis, we begin with a mechanics based model of a population of cells where individual cells are represented as deformable spheres. We apply growth, and when cells exceed a threshold size they divide according to some probability distribution defined by angle $\phi$, illustrated in Fig. 1 b. Our main quantitative simulation result is visualized in Fig. 11d, where the ellipses are visualizations of the average growth tensor for a simulated cell population. In this paper, we chose to study two different distributions of $\phi$ inspired by compelling scenarios from the literature. First, based on the observations in (Théry et al. 2007) and (Minc et al. 2011), where cell shape is tightly controlled, we note that $\phi$ is predicted correctly by theory only when some level of gaussian noise is included in the theoretical model. This motivates us to test a wrapped normal distri-
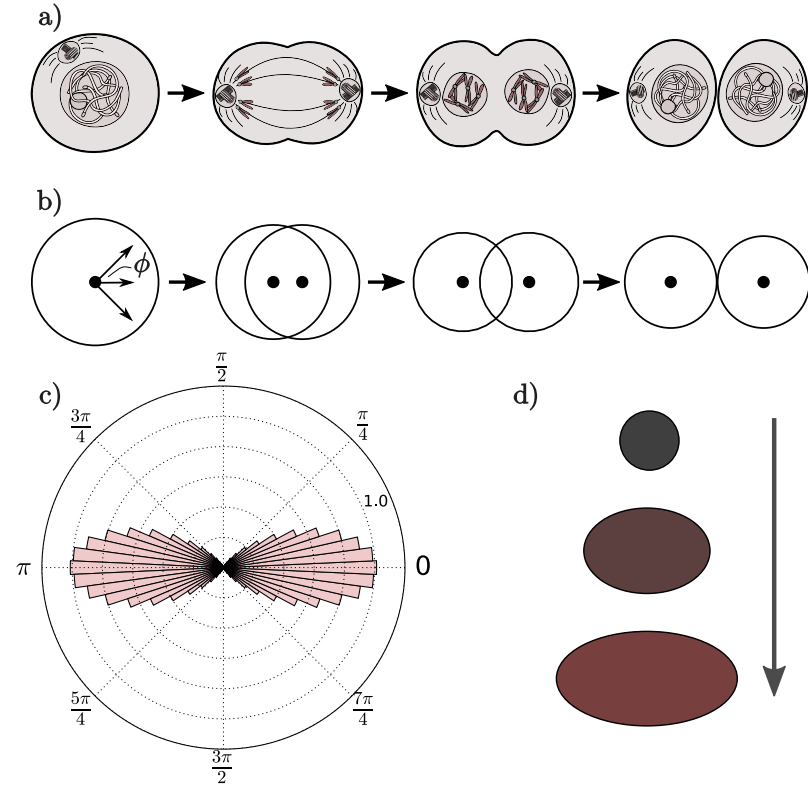

d)

Figure 1: Biological cells undergo cell division (a) where a single cell splits into two cells; A discrete model of a dividing cell (b) defines division orientation by some angle $\phi$; The angle $\phi$ is a random variable defined by a prescribed probability distribution where $\phi$ is equivalent to $\phi+\pi$ (c); As a population of cells grows, tracking the relative positions of each cell can be used to describe the resulting morphology (d).

bution of $\phi$ in order to understand how the level of noise will impact morphogenesis. Second, based on the observation in (Williams et al. 2014), that cells in genetically normal epidermal sheets undergo almost exclusively planar and perpendicular division, we are motivated to study a binomial distribution of $\phi$. With this binomial distribution with two perpendicular modes, we test how changing the probability of each mode will influence morphogenesis. Using our framework, $\phi$ as a function of many division angle determining rules beyond these two examples can be studied.

The remainder of the paper is organized as follows. We begin in Section 2 by describing our methods for simulating cell population growth and division, the specifics of the division angle distributions that we test, and our method for computing the average growth induced deformation from our stochastic simulations. In Section 3, we report and interpret our 
simulation results. Finally, we conclude in Section 4 .

\section{Methods}

Theoretical and computational modeling is a powerful tool for understanding biological systems (Ambrosi et al. 2011; Taber, 1995). In particular, modeling can be used to connect cellular-scale mechanisms with events on the tissue scale (Lowengrub et al. 2010, Stolarska et al., 2009) and understand complex systems in vivo (Di Ventura et al., 2006). For example, computational models can be used to study changes in biological tissue during development (Giorgi et al., 2014, Lejeune et al., 2016), the progression of disease in the lungs (Eskandari et al. 2016) and cardiovascular system (Göktepe et al.|, 2010||Zohdi et al., 2004), tumor growth and progression towards cancer (Frieboes et al., 2010; Wise et al. 2008), and wound healing (Tepole and Kuhl, 2016, Tepole 2016). Computational modeling can also be used to study fundamental mechanisms controlling tissue growth and cellular organization such as growth suppressing contact inhibition (Galle et al. 2009), monolayer growth and formation (Byrne and Drasdo, 2008; Galle et al. 2006), the relationship between stress and growth (Ambrosi et al. 2012), and differential adhesion (Hogeweg, 2000). In this paper, we use a computational model of a population of unconstrained growing and dividing cells to specifically investigate the influence of division angle on population morphology.

We begin this section by describing the formulation of our mechanics based model in Section 2.1. Using our computational model, we can investigate the influence of many different rules for determining cell division angle. Here we investigate two different distributions, a wrapped normal distribution and a binomial distribution, detailed in Section 2.2. Finally, we describe a method for quantitatively comparing the results of multiple stochastic simulations in section 2.3 where we describe how to compute the approximate average deformation for each simulation.

\subsection{Discrete mechanics-based computational model}

To study the influence of cell division angle on cell population morphology, we rely on a mechanics based

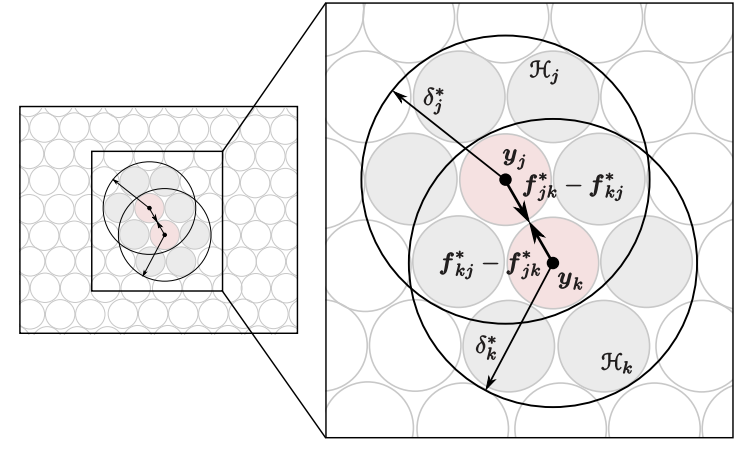

Figure 2: This figure illustrates nodes $\mathrm{j}$ and $\mathrm{k}$, with positions $\boldsymbol{y}_{\mathrm{j}}$ and $\boldsymbol{y}_{\mathrm{k}}$, horizon sizes $\delta_{\mathrm{j}}^{*}$ and $\delta_{,}^{*}{ }_{k}$, and horizons $\mathcal{H}_{\mathrm{j}}$ and $\mathcal{H}_{\mathrm{k}}$. The force densities at each node, $\boldsymbol{f}$, are computed based on the constitutive law formulated with eqns. 6.15 . At equilibrium, eqn. 5 is satisfied and total force density at each node is equal to zero.

computational framework where each cell is represented discretely by a central node. For our computational framework, we use peridynamics, a method which formulates the classical balance equations in integral form (Silling, 2000; Silling et al., 2007, Ren et al., 2016). Specifically, we use a version of state based dual-horizon peridynamics adapted for biological growth and cell division (Lejeune and Linder. 2017). In our model, cells grow and divide according to a prescribed growth law and cell division condition, and relax towards mechanical equilibrium iteratively via the peridynamic equation of motion.

We formulate the equation of motion and constitutive law at every node $\mathrm{j}$, where the index $\mathrm{k}$ refers to all of the other nodes that enter the equations centered at node $\mathrm{j}$. Here, the symbol $*$ indicates terms which are different from the traditional implementation of peridynamics because they accommodate cell growth and cell division. In the peridynamic equations of motion, we first define the concept of horizon $\mathcal{H}$. The horizon of node $\mathrm{j}, \mathcal{H}_{\mathrm{j}}$, is the domain within which nodes $\mathrm{k}$ will receive force exerted by node $\mathrm{j}$. The size of this domain is determined by parameter

$$
\delta_{\mathrm{i}}^{*}=\left(1+g_{\mathrm{i}}\right) \delta_{\mathrm{i}}^{0}
$$

for any node $\mathrm{i}$, where $g_{\mathrm{i}}$ is radial growth and $\delta_{\mathrm{i}}^{0}$ is an initial horizon size that will encompass the node's immediate neighbors. Using this definition of $\delta_{\mathrm{j}}$, the 
presence of node $\mathrm{k}$ in the horizon is determined as

$$
\mathcal{H}_{\mathrm{j}}=\left\{\mathrm{k} \mid \quad\left\|\boldsymbol{y}_{\mathrm{k}}-\boldsymbol{y}_{\mathrm{j}}\right\|<\delta_{\mathrm{j}}\right\}
$$

where $\boldsymbol{y}$ refers to nodal position in the current configuration. Because we are using dual-horizon peridynamics, we also define the dual-horizon of node $j$ as

$$
\mathcal{H}_{\mathrm{j}}^{\prime}=\left\{\mathrm{k} \mid \mathrm{j} \in \mathcal{H}_{\mathrm{k}}\right\}
$$

where all nodes $\mathrm{k}$ within $\mathcal{H}_{\mathrm{j}}^{\prime}$ exert a force on node j. In the peridynamic formulation, nodes only interact with other nodes within their horizon and dualhorizon (Ren et al., 2016). We also define the growth adjusted volume of a node $\mathrm{i}$ as

$$
\Delta V_{\mathrm{i}}^{*}=\left(1+g_{i}\right)^{n} \Delta V_{\mathrm{i}}
$$

where $n$ is the problem dimension, in this paper $n=$ 2. Using these quantities, we can define the quasistatic equation of motion in the discrete form as

$$
\begin{aligned}
0= & \sum_{\mathrm{k} \in \mathcal{H}_{\mathrm{j}}^{\prime}} \boldsymbol{f}_{\mathrm{jk}}^{*}\left(\boldsymbol{y}_{\mathrm{j}}, \boldsymbol{y}_{\mathrm{k}}\right) \Delta V_{\mathrm{k}}^{*}- \\
& \sum_{\mathrm{k} \in \mathcal{H}_{\mathrm{j}}} \boldsymbol{f}_{\mathrm{kj}}^{*}\left(\boldsymbol{y}_{\mathrm{j}}, \boldsymbol{y}_{\mathrm{k}}\right) \Delta V_{\mathrm{k}}^{*}
\end{aligned}
$$

where $\boldsymbol{f}_{\mathrm{jk}}^{*}$ is the force density exerted on $\mathrm{j}$ and $-\boldsymbol{f}_{\mathrm{kj}}^{*}$ is the reaction force density exerted on $\mathrm{j}$ due to force that $\mathrm{j}$ exerts on $\mathrm{k}$. Using adaptive dynamic relaxation (Kilic and Madenci, 2010), we solve this equation multiple times over the course of our simulation.

Next, we define the constitutive law needed to compute $\boldsymbol{f}^{*}$. Here, our constitutive law is linear elastic and includes a radial growth term $g$. We start by defining separation distance $\left\|\boldsymbol{\xi}_{\mathrm{jk}}^{*}\right\|$ that depends on the radius $r$ and growth $g$ of two adjacent nodes as

$$
\left\|\boldsymbol{\xi}_{\mathrm{jk}}^{*}\right\|=\left(1+g_{\mathrm{j}}\right) r_{\mathrm{j}}+\left(1+g_{\mathrm{k}}\right) r_{\mathrm{k}} \text {. }
$$

In the traditional implementation of peridynamics, $\boldsymbol{\xi}_{\mathrm{jk}}$ would refer to the vector connecting nodes in the initial configuration. Here, we define $\boldsymbol{\xi}_{\mathrm{jk}}^{*}$ as the "bond" between node $\mathrm{j}$ and node $\mathrm{k}$, where it only serves a notational purpose, intended to be analogous to the traditional implementation of peridynamics.

The first step to defining our constitutive relation is determining which nodes enter it. As a starting point, we know that nodes $\mathrm{k}$ outside the horizon and dual-horizon of node $\mathrm{j}$ will not contribute. Then, we determine which bonds are "broken" and which bonds are "unbroken" by checking the stretch between potentially interacting nodes. Using our definition for $\left\|\boldsymbol{\xi}_{\mathrm{jk}}^{*}\right\|$ given in eqn. (6), we define stretch between nodes as

$$
s^{*}=\frac{\left\|\boldsymbol{y}_{\mathrm{k}}-\boldsymbol{y}_{\mathrm{j}}\right\|-\left\|\boldsymbol{\xi}_{\mathrm{jk}}^{*}\right\|}{\left\|\boldsymbol{\xi}_{\mathrm{jk}}^{*}\right\|} .
$$

For every node within $\mathcal{H}_{j}$ we check if $s^{*}$ at load step $t$ is below a critical value $s_{\max }$ and define $\gamma^{*}$ as

$$
\gamma^{*}\left(\boldsymbol{\xi}_{\mathrm{jk}}^{*}, t\right)=\left\{\begin{array}{ll}
1 & \text { if } s^{*}<s_{\max } \\
0 & \text { otherwise }
\end{array} .\right.
$$

In this definition, previously broken bonds are allowed to re-form which is a realistic assumption for biological materials. A typical value of $s_{\max }$ used in simulation is 1.15 . Then, we define an influence function $\underline{\omega}^{*}\left\langle\boldsymbol{\xi}_{\mathrm{jk}}^{*}\right\rangle$ using state based notation (Silling et al. 2007 ) where $\underline{\omega}$ is defined for every bond $\xi_{j k}^{*}$ as

$$
\underline{\omega}^{*}\left\langle\boldsymbol{\xi}_{\mathrm{jk}}^{*}\right\rangle=\gamma^{*}\left(\boldsymbol{\xi}_{\mathrm{jk}}^{*}, t\right) .
$$

We introduce $\underline{\omega}^{*}$ as a term separate from $\gamma^{*}$ for consistency with the literature, and to note that other choices for influence function, such as one that decays exponentially with distance, are possible (Littlewood, 2015). Using our influence function, we define the weighted volume of the horizon at node $\mathrm{j}$ as

$$
m_{\mathrm{j}}^{*}=\sum_{\mathrm{k} \in \mathcal{H}_{\mathrm{j}}} \underline{\omega}^{*}\left\langle\boldsymbol{\xi}_{\mathrm{jk}}^{*}\right\rangle\left\|\boldsymbol{\xi}_{\mathrm{jk}}^{*}\right\|^{2} \Delta V_{\mathrm{k}}^{*}
$$

We define the extension state of bond $\boldsymbol{\xi}_{\mathrm{jk}}^{*}$ simply as

$$
\underline{e}^{*}\left\langle\boldsymbol{\xi}_{\mathrm{jk}}^{*}\right\rangle=\left\|\boldsymbol{y}_{\mathrm{k}}-\boldsymbol{y}_{\mathrm{j}}\right\|-\left\|\boldsymbol{\xi}_{\mathrm{jk}}^{*}\right\| \text {. }
$$

Then, using the extension state and weighted volume we determine the dilation at node $\mathrm{j}$ as

$$
\theta_{\mathrm{j}}^{*}=\frac{n}{m_{\mathrm{j}}^{*}} \sum_{\mathrm{k} \in \mathcal{H}_{\mathrm{j}}} \underline{\omega}^{*}\left\langle\boldsymbol{\xi}_{\mathrm{jk}}^{*}\right\rangle\left\|\boldsymbol{\xi}_{\mathrm{jk}}^{*}\right\| \underline{e}^{*}\left\langle\boldsymbol{\xi}_{\mathrm{jk}}^{*}\right\rangle \Delta V_{\mathrm{k}}^{*}
$$

where $n=2$ is the problem dimension. Using bond extension state and nodal dilation, we determine the deviatoric extension state for bond $\boldsymbol{\xi}_{\mathrm{jk}}^{*}$ at node $\mathrm{j}$ as

$$
\underline{e}^{d^{*}}\left\langle\boldsymbol{\xi}_{\mathrm{jk}}^{*}\right\rangle=\underline{e}^{*}\left\langle\boldsymbol{\xi}_{\mathrm{jk}}^{*}\right\rangle-\frac{\theta_{\mathrm{j}}^{*}\left\|\boldsymbol{\xi}_{\mathrm{jk}}^{*}\right\|}{n}
$$


Finally, we calculate the magnitude of bond force density acting on node $\mathrm{k}$ due to node $\mathrm{j}$ as

$$
\begin{aligned}
\underline{t}_{\mathrm{kj}}^{*}\left\langle\boldsymbol{\xi}_{\mathrm{jk}}^{*}\right\rangle= & \frac{n \kappa \theta_{\mathrm{j}}^{*}}{m_{\mathrm{j}}^{*}} \underline{\omega}^{*}\left\langle\boldsymbol{\xi}_{\mathrm{jk}}\right\rangle|| \boldsymbol{\xi}_{\mathrm{jk}}^{*} \|+ \\
& \frac{n(n+2) \mu}{m_{\mathrm{j}}^{*}} \underline{\omega}^{*}\left\langle\boldsymbol{\xi}_{\mathrm{jk}}\right\rangle \underline{e}^{d^{*}}\left\langle\boldsymbol{\xi}_{\mathrm{jk}}\right\rangle
\end{aligned}
$$

where $\underline{e}^{d *}$ is computed with $\theta_{j}^{*}$ and $\kappa$ and $\mu$ are Lamé parameters selected such that $\nu=0.45$ to capture the incompressibility of biological tissue while avoiding the numerical issues that arise when $\nu=0.5$ (Krischok and Linder, 2015). The magnitude of bond force density acting on node $\mathrm{j}$ due to node $\mathrm{k}, \underline{t}_{\mathrm{jk}}^{*}$, is computed with the same procedure. Using these magnitudes we compute $\boldsymbol{f}_{\mathrm{jk}}^{*}$ and $\boldsymbol{f}_{\mathrm{kj}}^{*}$ as

$$
\begin{aligned}
& \boldsymbol{f}_{\mathrm{jk}}^{*}\left(\boldsymbol{y}_{\mathrm{j}}, \boldsymbol{y}_{\mathrm{k}}\right)=t_{\mathrm{jk}}^{*} \cdot \frac{\boldsymbol{y}_{\mathrm{k}}-\boldsymbol{y}_{\mathrm{j}}}{\left\|\boldsymbol{y}_{\mathrm{k}}-\boldsymbol{y}_{\mathrm{j}}\right\|} \\
& \boldsymbol{f}_{\mathrm{kj}}^{*}\left(\boldsymbol{y}_{\mathrm{j}}, \boldsymbol{y}_{\mathrm{k}}\right)=t_{\mathrm{kj}}^{*} \cdot \frac{-\left(\boldsymbol{y}_{\mathrm{k}}-\boldsymbol{y}_{\mathrm{j}}\right)}{\left\|\boldsymbol{y}_{\mathrm{k}}-\boldsymbol{y}_{\mathrm{j}}\right\|} .
\end{aligned}
$$

With the constitutive relation defined, we are able to solve eqn. (5).

In addition to growing (increase in cell radius), the cells in this simulation must be able to divide when they exceed a threshold size defined as $g_{\max }=\sqrt{2}-1$ in $n=2$. We define $g_{0}$ as the growth of the parent cell and compute $g_{d}$, growth of each of the two daughter cells, as

$$
g_{d}=\sqrt[2]{\frac{1}{2}}\left(1+g_{0}\right)-1
$$

Cell division angle $\phi$, discussed further in Section 2.2 , defines unit vector $\boldsymbol{m}$. After division, the location of daughter cells $\mathrm{j}$ and $\mathrm{k}$ are defined as

$$
\begin{aligned}
\boldsymbol{y}_{\mathrm{j}} & =\boldsymbol{y}_{0}+\left(1+g_{d}\right) r_{0} \boldsymbol{m} \\
\boldsymbol{y}_{\mathrm{k}} & =\boldsymbol{y}_{0}-\left(1+g_{d}\right) r_{0} \boldsymbol{m} .
\end{aligned}
$$

For better numerical stability and potentially closer results to reality, division may also be carried out in a multi-step process, illustrated in Fig. 1. As a multi-step process, separation distance between dividing cells is increased over several steps and $g_{d}$ is altered at each step such that the area of both daughter cells minus the area of overlap remains constant.
And, for better numerical stability, the cells are prevented from dividing in unison. Our treatment of cell division is similar to other techniques defined in the literature (Drasdo and Höhme, 2005, Drasdo and Loeffler, 2001, Kreft et al., 2001). After each cell division step, the peridynamic framework is used to bring the system back to mechanical equilibrium.

\subsection{Probability distribution of the division angle}
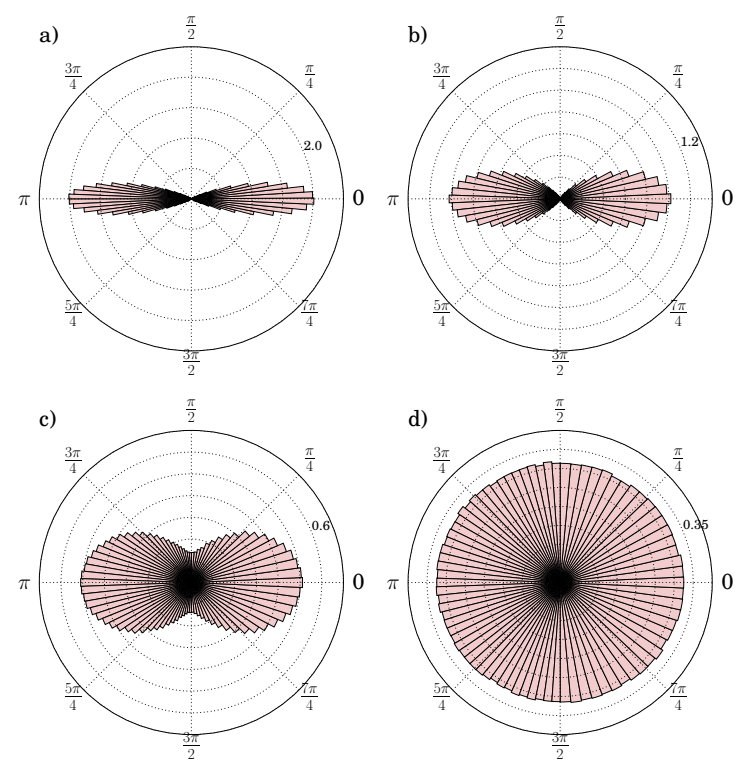

Figure 3: When cells divide, they divide on some axis determined by angle $\phi$. The angle $\phi$ is a random variable defined by a probability distribution. The circular histograms illustrate wrapped normal distributions where $\phi$ is equivalent to $\phi-\pi$. In all plots, the distribution of $\phi$ is defined by eqn. 19, the mean is 0 and $\beta$ in (a) is $\pi / 16$, in (b) is $\pi / 8$, in (c) is $\pi / 4$ and in (d) is $\pi / 2$. In (d), the value of $\beta$ is sufficiently large such that the distribution approximates a circular uniform distribution.

When cells divide, they do so at a given orientation, defined simply in 2D by angle $\phi$. Hypothetically, any probability distribution suitable for circular coordinates could be used to define $\phi$, and $\phi$ could be dependent on any global or local parameters and conditions. Here, we examine two simple cases where $\phi$ is defined by a probability distribution and every time a cell divides during the simulation $\phi$ is computed as an independent random variable sampled from the 
chosen probability distribution. In the first case that we test, $\phi$ is defined by a wrapped normal distribution, where the probability density function of the unwrapped distribution is

$$
f\left(x \mid \mu, \sigma^{2}\right)=\frac{1}{\sqrt{2 \sigma^{2} \pi}} e^{-\frac{(x-\mu)^{2}}{2 \sigma^{2}}}
$$

with mean $\mu$ and standard deviation $\sigma$. To convert a random variable sampled from this distribution, $\mathcal{N}(\mu, \sigma)$ we define $\phi$ as a function of the standard normal distribution

$$
\begin{gathered}
\phi=\operatorname{rem}(0.0+\beta \mathcal{N}(0,1), 2 \pi) \\
\phi:= \begin{cases}\phi & \text { if }-\pi / 2<\phi \leq \pi / 2 \\
\phi-\pi & \text { if } \pi / 2<\phi \\
\phi+\pi & \text { if } \phi \leq-\pi / 2 .\end{cases}
\end{gathered}
$$

The results of generating random variables using this procedure are visualized in Fig. 3 where histograms of $\phi$ and the equivalent $\phi-\pi$ are plotted at different levels of $\beta$. As $\beta \rightarrow \infty$, the distribution of $\phi$ approaches a circular uniform distribution, although for practical purposes values of $\beta$ arround $\pi / 2$ are sufficiently large such that the distribution is approximately circular uniform, as illustrated in Fig. 3d.

For the second case, $\phi$ is defined by a binomial random variable where we instead define $\beta$ as the probability that $\phi=\phi_{1}$ and $1-\beta$ as the probability that $\phi=\phi_{2}$. To determine $\phi$, we sample a uniform random variable in the range $0-1$, written as $\mathcal{U}(0,1)$ and determine $\phi$ as

$$
\phi= \begin{cases}\phi_{1} & \text { if } \mathcal{U}(0,1)<\beta \\ \phi_{2} & \text { if } \mathcal{U}(0,1) \geq \beta\end{cases}
$$

For the results presented in Section 3.1 $\phi_{1}=\pi / 2$ and $\phi_{2}=0$. As with the previous distribution, $\phi$ is wrapped around the circle and $\phi$ is equivalent to $\phi-\pi$, specifically $\phi=0$ is equivalent to $\phi=\pi$ and $\phi=\pi / 2$ is equivalent to $\phi=-\pi / 2$. The results of testing these two probability distributions for $\phi$ are presented in Section 3 .
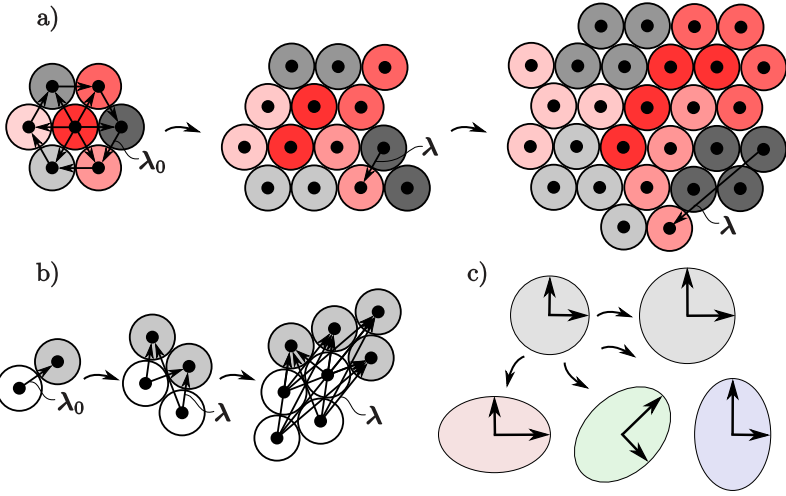

Figure 4: As illustrated in (a) the number of cells increases over the course of the simulation. In (a), each cell is color coded to match the cell that it originated from. The vector $\boldsymbol{\lambda}_{0}$ shown in (b) is the distance between two cells at the start of the simulation. Each vector $\boldsymbol{\lambda}_{0}$ corresponds to $4^{n} \boldsymbol{\lambda}$ vectors also shown in (b) where $n$ is the number of cell division events that have occurred. By relating $\boldsymbol{\Lambda}_{0}$ and $\boldsymbol{\Lambda}$ through eqn EQN, it is possible to compute an average deformation of the material defined by $\boldsymbol{F}$. In (c), examples of possible shapes of $\boldsymbol{F}$ are shown. An isotropic $\boldsymbol{F}$ is visualized as a circle.

\subsection{Computing average deformation}

In continuum models of biological systems, volumetric growth is typically described by growthinduced deformation gradient Ambrosi and Mollica. 2002, Ambrosi and Preziosi, 2008, Muñoz et al., 2007. Harvard Rodriguez et al., 1994, Zöllner et al., 2012). When growth is homogeneous and the system is neither externally loaded nor constrained, the deformation gradient and the growth-induced deformation gradient are identical. He we define an analogy to deformation gradient, which we call average deformation $\boldsymbol{F}$, that we use to interpret our simulation results. Being able to summarize the results of a single simulation with $\boldsymbol{F}$, which is a $2 \times 2$ matrix in two dimensions, facilitates the quantitative comparison of thousands of simulations. We compute average deformation $\boldsymbol{F}$ by tracking the relative position of cell centers. At the start of the simulation, cells are connected by $n$ stretch vectors $\lambda_{0}^{i}$ where $i=\{1 \ldots n\}$, illustrated in Fig. 4 a. Over the course of the simulation, the number of cells increases during every division event. Figure $4 \mathrm{~b}$ illustrates a single $\boldsymbol{\lambda}_{0}$ vector initially connecting cell $\mathrm{j}$ and cell $\mathrm{k}$, that after $m$ divi- 
sion events corresponds to a set of vectors connecting the $2^{m}$ daughter cells of $\mathrm{j}$ to the $2^{m}$ daughter cells of $\mathrm{k}$. This means that a single vector in the initial configuration corresponds to $2^{m} \times 2^{m}=4^{m}$ vectors in the current configuration. Therefore, for each vector $\boldsymbol{\lambda}_{0^{i}}$ that connects two cells at the start of the simulation, there will be $p=4^{m}$ current configuration $t$ vectors $\boldsymbol{\lambda}_{t^{i}}^{q}$ where $q=\{1 \ldots p\}$.

To compute the average deformation $\boldsymbol{F}$, we define an array of initial stretch vectors $\boldsymbol{\Lambda}_{0}$ as

$$
\begin{aligned}
\boldsymbol{\Lambda}_{0}= & {\left[\boldsymbol{\lambda}_{0_{1}}^{1} \lambda_{0_{1}}^{2} \ldots \lambda_{0_{1}}^{p}\right.} \\
& \left.\boldsymbol{\lambda}_{0_{2}}^{1} \boldsymbol{\lambda}_{0_{2}}^{2} \ldots \lambda_{0_{2}}^{p} \ldots \ldots \lambda_{0_{n}}^{1} \lambda_{0_{n}}^{2} \ldots \lambda_{0_{n}}^{p}\right] .
\end{aligned}
$$

The vectors $\lambda_{0_{i}}$ will each be repeated $p$ times with $n \times p$ total vectors making up $\boldsymbol{\Lambda}_{0}$. Then we define an array of current stretch vectors $\boldsymbol{\Lambda}_{t}$ as

$$
\begin{aligned}
\boldsymbol{\Lambda}_{t}= & {\left[\boldsymbol{\lambda}_{t_{1}}^{1} \lambda_{t_{1}}^{2} \ldots \lambda_{t_{1}}^{p}\right.} \\
& \left.\boldsymbol{\lambda}_{t_{2}}^{1} \boldsymbol{\lambda}_{t_{2}}^{2} \ldots \lambda_{t_{2}}^{p} \ldots \ldots \lambda_{t_{n}}^{1} \lambda_{t_{n}}^{2} \ldots \lambda_{t_{n}}^{p}\right] .
\end{aligned}
$$

Given these arrays, we can define average deformation in the current configuration $\boldsymbol{F}_{t}$ with the equation

$$
\boldsymbol{F}_{t} \boldsymbol{\Lambda}_{0}=\boldsymbol{\Lambda}_{t}
$$

To solve this over-determined system of equations (in $2 \mathrm{D} \boldsymbol{F}_{t}$ is a $2 \times 2$ matrix and $\boldsymbol{\lambda}$ is a $2 \times 1$ vector), we use the normal equation

$$
\boldsymbol{F}_{t}=\boldsymbol{\Lambda}_{t} \boldsymbol{\Lambda}_{0}^{T}\left(\boldsymbol{\Lambda}_{0} \boldsymbol{\Lambda}_{0}^{T}\right)^{-1} .
$$

In Fig. 4t, $\boldsymbol{F}_{t}$ is visualized as an ellipse. The orientation and scale of the ellipse are determined by the principal stretches (eigenvalues) and principal directions (eigenvectors) of $\boldsymbol{F}_{t}$. The computed value of $\boldsymbol{F}_{t}$ is used to quantify the simulation results presented in Section 3. When $\boldsymbol{F}$ is written rather than $\boldsymbol{F}_{t}$, it refers to average deformation at the end of the simulation, $t=t_{\text {final }}$.

\section{Results and Discussion}

The main outcome of simulating cell populations with the cell division angle probability distributions defined in Section 2.2 is an understanding of two potential mechanisms controlling population morphology. In Section 3.1, we show that the degree of

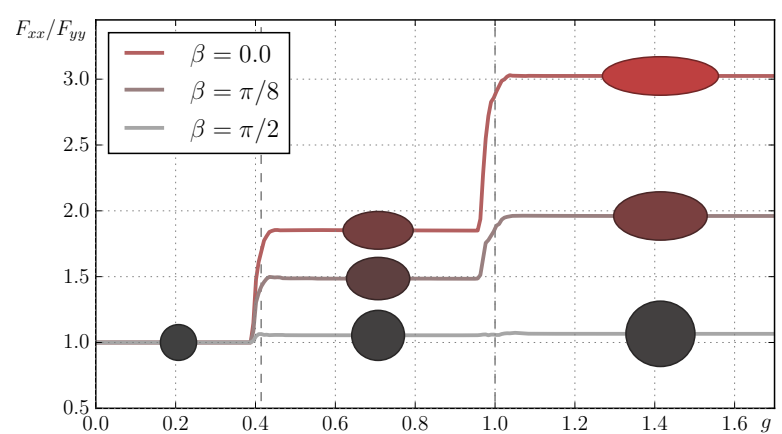

Figure 5: For populations of cells that divide according to $\phi$ defined by the distribution illustrated in Fig. 3 where $\phi=0.0+\beta \mathcal{N}(0,1)$ wrapped, population morphology is influenced by $\beta$. In this plot, the ratio $F_{x x} / F_{y y}$ is plotted with respect to $\beta$ where each line shows the average of 10 simulations. The ellipses superimposed on the plot illustrate the principal components and directions of $\boldsymbol{F}$, and the dashed vertical lines show cell division events. Morphology changes as $g$ increases and more cell division events occur. By comparing $\beta=0, \beta=\pi / 8$, and $\beta=\pi / 2$ it is clear that low values of $\beta$ result in more anisotropy.

anisotropy in macroscale deformation can be controlled by the amount of noise present in determining $\phi$. In Section 3.1, we show an alternative mechanism for controlling cell population morphology through a binomial distribution of $\phi$.

\subsection{Wrapped normal distribution}

Given the distribution of $\phi$ defined in eqn. 19 and illustrated in Fig. 3, we aim to understand how the level of noise $\beta$ influences population morphology. Because $\phi$ is a random variable, simulation results are stochastic. Therefore, at a given value of $\beta$ we perform multiple simulations. To quantify simulation results, we use the metric $\boldsymbol{F}$, defined in Section 2.3 For each set of input variables, we run multiple stochastic simulations and report the simulation results as the components of $\boldsymbol{F}$ averaged over multiple runs. For all the simulations in this section, there is an initial population of 37 nodes equally spaced in a hexagonal configuration. In Fig. 5, we compare three different values of $\beta, \beta=0.0, \beta=\pi / 8$ and $\beta=\pi / 2$ by plotting the ratio of $F_{x x}$ to $F_{y y}$, averaged over ten simulations. The ellipses in Fig. 5 are visualizations 


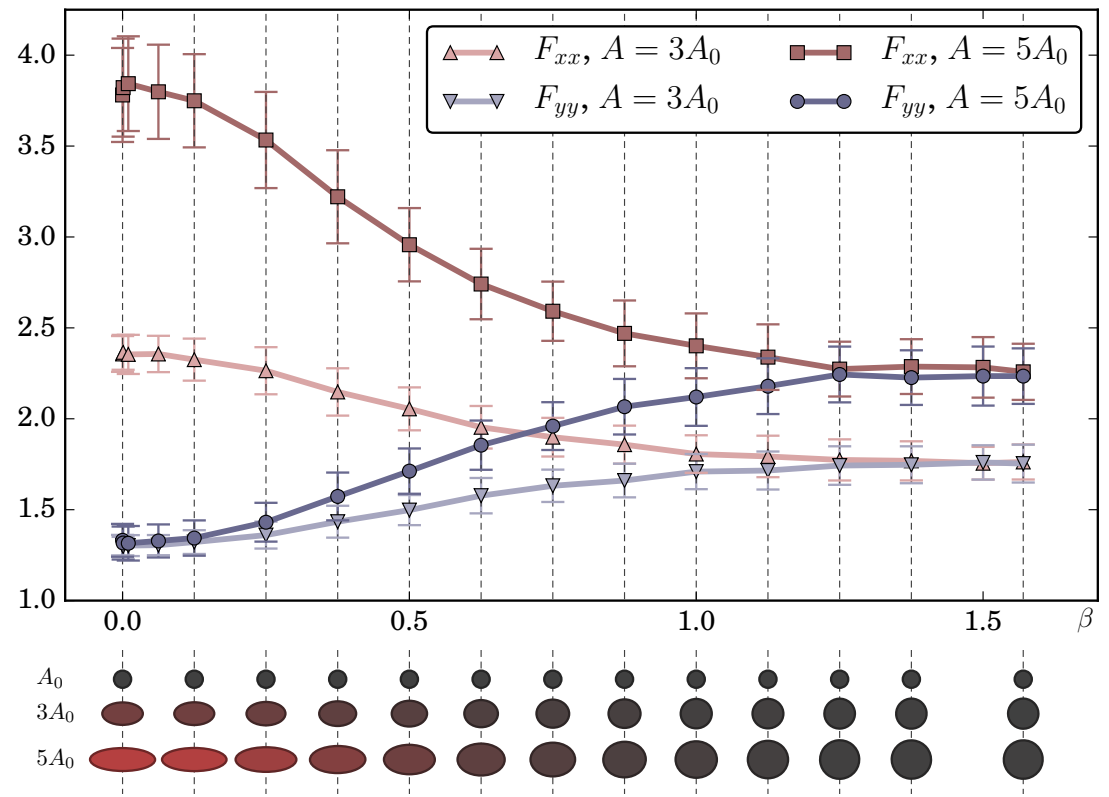

Figure 6: Population morphology can be controlled by the amount of noise present in the cell division angle. The level of noise is represented by $\beta$, where $\phi=0.0+$ $\beta \mathcal{N}(0,1)$ wrapped. At low levels of noise, $\beta \approx 0$, growth will be highly anisotropic. As $\beta$ increases and the distribution of $\phi$ becomes more uniform, growth becomes isotropic. The amount of anisotropy in growth is quantified by examining the components of $\boldsymbol{F}$ and visualized by plotting $\boldsymbol{F}$ as an ellipse. For each set of ellipses, the upper row shows $A=$ $A_{0}$, the middle row shows $A=3 A_{0}$ and the lower row shows $A=5 A_{0}$ where $A_{0}$ is area at the start of the simulation. Each point on the plot represents the average of 200 simulations, the error bars indicate one standard deviation, and the offdiagonal components of $\boldsymbol{F}, F_{x y}$ and $F_{y x}$, are both $\approx 0$.

of $\boldsymbol{F}$ corresponding to the point on the line where they are plotted. The results when plotting $\beta=0.0$ and $\beta=\pi / 2$ confirm intuition: when $\beta$ is simply a fixed value, population growth is highly anisotropic and when $\beta=\pi / 2$ the nearly uniform distribution of $\phi$ results in isotropic population growth. For the case where $\beta=\pi / 8$, the simulation results show an intermediate degree of anisotropy. In order to better investigate this intermediate regime of $\beta$, we conduct a parameter sweep.

Figure 6 shows hows varying $\beta$ influences the components of $\boldsymbol{F}$. We plot the components $F_{x x}$ and $F_{y y}$ with respect to $\beta$ at two different levels of growth averaged over 200 simulations at each level of $\beta$. When area growth $A=3 A_{0}$, with $A_{0}$ equal to initial area, each cell divides once by the end of the simulation. When $A=5 A_{0}$, each cell divides twice. Below the graph, we show the corresponding visual representations of $\boldsymbol{F}$ starting with the initial configuration when $A=A_{0}$. For all values of $\beta$ the components of $\boldsymbol{F}$ when $A=A_{0}$ are $F_{x x}=F_{y y}=1.0$. And, in all cases, the off-diagonal components of $\boldsymbol{F}$ are approximately 0 . From these results, we can see that at low levels of $\beta, \boldsymbol{F}$ is highly anisotropic. When $\beta \approx 0$, the value of $F_{y y}$ remains close to $\sqrt{2}$, which is the value of $F_{y y}$ prior to the occurrence of the first division event. Furthermore, small values of $\beta$ do not significantly reduce the anisotropy as the curves are relatively flat when $\beta$ is small. For sufficiently high $\beta$, approximatly $1.25<\beta$, the components $F_{x x}$ and $F_{y y}$ are approximately equal and therefore growth is isotropic. This is consistent with the observation that at this level of $\beta$, the distribution of $\phi$ approaches a circular normal distribution, as illustrated in Fig. 3. For the approximate range $0.05<\beta<1.25$ growth is anisotropic and dependent on $\beta$ : as the amount of noise increases, anisotropy decreases. And, by comparing the results when $A=3 A_{0}$ and $A=5 A_{0}$ we can clearly see that more division events allow for more anisotropy to arise. A (relatively crude) approximate method for determining the components of $\boldsymbol{F}$ without conducting any simulations would be to draw a line from $F_{x x}=A /(\sqrt{2})$ at $\beta=0.05$ to $F_{x x}=\sqrt{A}$ at $\beta=1.25$ and find $F_{x x}$ at a given value of $\beta$ using the approximate fit. Then, $F_{y y}$ would be computed as $F_{y y}=A / F_{x x}$. From these simulations, the main result is that we can connect noise in $\phi$, a cellular scale contribution, to macroscale growth. This is im- 


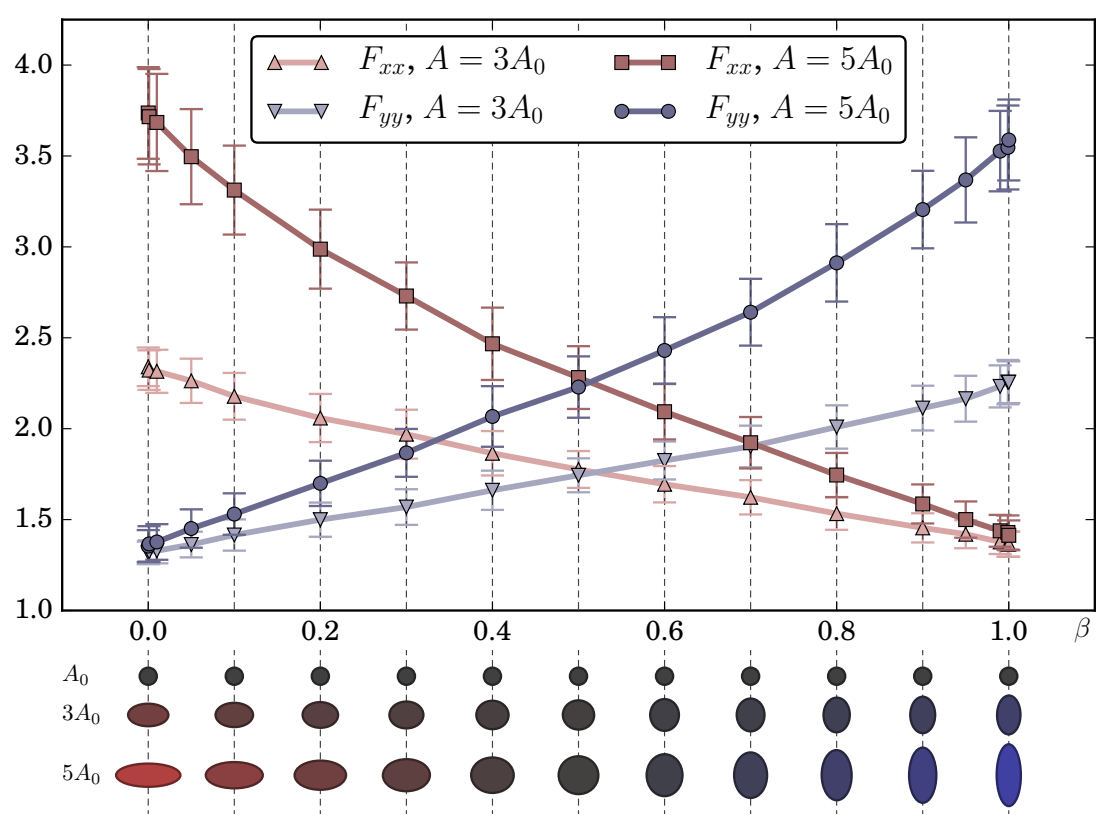

Figure 7: An alternative path to controlling cell population morphology is though multiple potential division angles. Here, $\phi$ is determined by a binomial distribution where the probability of $\phi=\pi / 2$ is $\beta$ and the probability of $\phi=0$ is $1-\beta$. When $\beta=0$ growth is anisotropic with the $\phi=0$ direction dominant, when $\beta=0.5$ growth is isotropic, and when $\beta=1$ growth is anisotropic with the $\phi=\pi / 2$ direction dominant. The amount of anisotropy in growth is quantified by examining the components of $\boldsymbol{F}$ and visualized by plotting $\boldsymbol{F}$ as an ellipse. For each set of ellipses, the upper row shows $A=$ $A_{0}$, the middle row shows $A=3 A_{0}$ and the lower row shows $A=5 A_{0}$ where $A_{0}$ is area at the start of the simulation. Each point on the plot represents the average of 200 simulations, the error bars indicate one standard deviation, and the offdiagonal components of $\boldsymbol{F}, F_{x y}$ and $F_{y x}$, are both $\approx 0$. portant because is shows a pathway between internal and external cues that influence noise in biological processes and morphogenesis.

\subsection{Binomial distribution}

An alternative mechanism for controlling morphogenesis through $\phi$ emerges when we study the distribution defined in eqn. 20. Similar to the approach taken for the results presented in Fig. 6. we have the distribution from eqn. 20 defined in terms of parameter $\beta$ where varying $\beta$ controls the division angle. In Fig. 7, we plot the components $F_{x x}$ and $F_{y y}$ with respect to $\beta$ at $A=3 A_{0}$ and $A=5 A_{0}$. Each point on the plot represents the average value of 200 simulations. Below the graph, we show the corresponding visual representations of $\boldsymbol{F}$. Once again, for all values of $\beta$ the components of $\boldsymbol{F}$ when $A=A_{0}$ are $F_{x x}=F_{y y}=1.0$. And, in all cases, the off-diagonal components of $\boldsymbol{F}$ are approximately 0 . From the results illustrated in Fig. 7, it is clear that at one extreme, where $\beta=0$, growth will be highly anisotropic, with $F_{x x}$ exceeding $F_{y y}$. Identical to the results seen in Section $3.1 F_{y y} \approx \sqrt{2}$ at this extreme. At the other extreme, with $\beta=1.0$, the opposite is true: $F_{y y}$ exceeds $F_{x x}$. This is entirely consistent with intuition, because fundamentally in both cases $\beta$ is just set equal to some fixed value. In the center of the graph, where $\beta=0.5$, growth is isotropic with $F_{x x}=F_{y y}=\sqrt{A}$. And, it is clear that by tuning $\beta$, i.e. the respective probabilities of each fixed division angle, the degree and direction of anisotropy in macroscale growth can be tightly controlled. A fairly accurate and approximate method for estimating results without conducting simulations could be conducted by defining $F_{x x}$ as a line connecting the point $\left(\beta=0, F_{x x}=A / \sqrt{2}\right)$ to the point $\left(\beta=1.0, F_{x x}=\sqrt{2}\right)$, solving for $F_{x x}$ from $\beta$ and computing $F_{y y}$ as $F_{y y}=A / F_{x x}$. By investigating the binary distribution of $\phi$, we have shown another mechanism by which cell population morphology can be controlled. This scenario is an important test case for beginning to understand the implications of experimentally observed bimodal distributions of $\phi$. 


\section{Conclusion}

The main objective of this work was to explore how the probability distribution of the cell division angle influences cell population morphology. Our results indicate that the underlying distribution dictating the division angle controls the direction and degree of anisotropy on the population scale. In Section 3.1 we demonstrate that the level of noise entering the division angle controls average population growth, with more noise decreasing the degree of anisotropy in a consistent manner. This knowledge can be used to interpret experimental studies reporting the preferred division angle of a single cell: given some mean and standard deviation of single cell division angle, we can use our results to make predictions about the expected population morphology. In Section 3.1 we show that varying the probability of two possible division angles is an alternative potential mechanism for tuning morphology that may be relevant for planar cell structures such as epithelia. This mechanism may also be particularly relevant for studying cells that tend to divide either parallel or perpendicular to some chemical gradient or external load.

It is also worth mentioning that the technique presented in Section 2.3 is general to any discrete model of cells, it does not have to be applied to the one presented in Section 2.1 or use the growth and division laws presented in Section 2.2 For a given cell type, the computational model used can be as detailed and specific as is feasible and realistic. This work is only just the beginning of serious consideration of the macroscale implications of the probability distribution of cell division angle. Future work with this framework may address topics such as alternative growth and division laws, how individual cell anisotropy may influence macroscale growth, or how cell division behavior may connect to other forms of mechanically driven emergent behavior such as buckling and wrinkling (Dortdivanlioglu et al. 2016). Overall, our results indicate that the distribution of cell division angle emerges as a critical factor in morphogenesis and presents a basis for further investigation of the topic.

\section{Acknowledgements}

This work was supported by the National Science Foundation Graduate Research Fellowship under Grant No. DGE-114747.

\section{References}

Akanuma, T., Chen, C., Sato, T., Merks, R., Sato, T., 2016. Memory of cell shape biases stochastic fate decision-making despite mitotic rounding. $\mathrm{Na}-$ ture Communications 7.

Ambrosi, D., Ateshian, G., Arruda, E., Cowin, S., Dumais, J., Goriely, A., Holzapfel, G., Humphrey, J., Kemkemer, R., Kuhl, E., Olberding, J., Taber, L., Garikipati, K., 2011. Perspectives on biological growth and remodeling. Journal of the Mechanics and Physics of Solids 59, 863-883.

Ambrosi, D., Mollica, F., 2002. On the mechanics of a growing tumor. International Journal of Engineering Science 40, 1297-1316.

Ambrosi, D., Preziosi, L., 2008. Cell adhesion mechanisms and stress relaxation in the mechanics of tumours. Biomech Model Mechanobiol 8, 397-413.

Ambrosi, D., Preziosi, L., Vitale, G., 2012. The interplay between stress and growth in solid tumors. Mechanics Research Communications 42, 87-91.

Bosveld, F., Markova, O., Guirao, B., Martin, C., Wang, Z., Pierre, A., Balakireva, M., Gaugue, I., Ainslie, A., Christophorou, N., Lubensky, D., 2016. Epithelial tricellular junctions act as interphase cell shape sensors to orient mitosis. Nature .

Byrne, H., Drasdo, D., 2008. Individual-based and continuum models of growing cell populations: a comparison. Journal of Mathematical Biology 58, 657-687.

Corrigan, A., Shrestha, R., Draviam, V., Donald, A., 2015. Modeling of noisy spindle dynamics reveals separable contributions to achieving correct orientation. Biophysical journal 109, 1398-1409. 
Di Ventura, B., Lemerle, C., Michalodimitrakis, K., Serrano, L., 2006. From in vivo to in silico biology and back. Nature 443, 527-533.

Dortdivanlioglu, B., Javili, A., Linder, C., 2016. Computational aspects of morphological instabilities using isogeometric analysis. Computer Methods in Applied Mechanics and Engineering 10.1016/j.cma.2016.06.028.

Drasdo, D., Höhme, S., 2005. A single-cell-based model of tumor growth in vitro: monolayers and spheroids. Physical Biology 2, 133-147.

Drasdo, D., Loeffler, M., 2001. Individual-based models to growth and folding in one-layered tissues: intestinal crypts and early development. Nonlinear Analysis: Theory, Methods \& Applications 47, $245-256$.

Eskandari, M., Javili, A., Kuhl, E., 2016. Elastosis during airway wall remodeling explains multiple co-existing instability patterns. Journal of theoretical biology 403, 209-218.

Frieboes, H., Jin, F., Chuang, Y., Wise, S., Lowengrub, J., Cristini, V., 2010. Three-dimensional multispecies nonlinear tumor growth-ii: tumor invasion and angiogenesis. Journal of theoretical biology 264, 1254-1278.

Galle, J., Aust, G., Schaller, G., Beyer, T., Drasdo, D., 2006. Individual cell-based models of the spatial-temporal organization of multicellular systems-achievements and limitations. Cytometry Part A 69.

Galle, J., Preziosi, L., Tosin, A., 2009. Contact inhibition of growth described using a multiphase model and an individual cell based model. Applied Mathematics Letters 22, 1483-1490.

Gibson, W., Veldhuis, J., Rubinstein, B., Cartwright, H., Perrimon, N., Brodland, G., Nagpal, R., Gibson, M., 2011. Control of the mitotic cleavage plane by local epithelial topology. Cell 144, 427-438.

Gillies, T.E., Cabernard, C., 2011. Cell division orientation in animals. Current Biology 21, R599 R609.
Giorgi, M., Carriero, A., Shefelbine, S., Nowlan, N., 2014. Mechanobiological simulations of prenatal joint morphogenesis. Journal of biomechanics 47, 989-995.

Göktepe, S., Abilez, O., Parker, K., Kuhl, E., 2010. A multiscale model for eccentric and concentric cardiac growth through sarcomerogenesis. Journal of theoretical biology 265, 433-442.

Harvard Rodriguez, E., Hoger, A., McCulloch, A., 1994. Stress-dependent finite growth in soft elastic tissues. Journal of biomechanics 27, 455-467.

Hogeweg, P., 2000. Evolving mechanisms of morphogenesis: on the interplay between differential adhesion and cell differentiation. Journal of Theoretical Biology 203, 317-333.

Juschke, C., Xie, Y., Postiglione, M.P., Knoblich, J.A., 2013. Analysis and modeling of mitotic spindle orientations in three dimensions. Proceedings of the National Academy of Sciences 111, 1014-1019.

Kilic, B., Madenci, E., 2010. An adaptive dynamic relaxation method for quasi-static simulations using the peridynamic theory. Theoretical and Applied Fracture Mechanics 53, 194-204.

Kreft, J., Picioreanu, C., Wimpenny, J., van Loosdrecht, M., 2001. Individual-based modelling of biofilms. Microbiology 147, 2897-2912.

Krischok, A., Linder, C., 2015. On the enhancement of low-order mixed finite element methods for the large deformation analysis of diffusion in solids. International Journal for Numerical Methods in Engineering 106, 278-297.

Lamb, B., Luo, W., Nagdas, S., Yousaf, M., 2014. Cell division orientation on biospecific peptide gradients. ACS Applied Materials \& Interfaces 6, $11523-11528$.

Lejeune, E., Javili, A., Weickenmeier, J., Kuhl, E., Linder, C., 2016. Tri-layer wrinkling as a mechanism for anchoring center initiation in the developing cerebellum. Soft matter 12, 5613-5620. 
Lejeune, E., Linder, C., 2017. Modeling tumor growth with peridynamics. submitted for publication .

Littlewood, D., 2015. Roadmap for peridynamic software implementation. SAND Report, Aandia National Laboratories, Albuquerque, NM and Livermore, CA. .

Lowengrub, J.S., Frieboes, H.B., Jin, F., Chuang, Y.L., Li, X., Macklin, P., Wise, S.M., Cristini, V., 2010. Nonlinear modelling of cancer: bridging the gap between cells and tumours. Nonlinearity 23, R1-R91.

Matamoro-Vidal, A., Salazar-Ciudad, I., Houle, D. 2015. Making quantitative morphological variation from basic developmental processes: Where are we? the case of the drosophila wing. Developmental Dynamics 244, 1058-1073.

Minc, N., Burgess, D., Chang, F., 2011. Influence of cell geometry on division-plane positioning. Cell $144,414-426$.

Minc, N., Piel, M., 2012. Predicting division plane position and orientation. Trends in cell biology 22, 193-200.

Muñoz, J., Barrett, K., Miodownik, M., 2007. A deformation gradient decomposition method for the analysis of the mechanics of morphogenesis. Journal of biomechanics 40, 1372-1380.

Nestor-Bergmann, A., Goddard, G., Woolner, S., 2014. Force and the spindle: Mechanical cues in mitotic spindle orientation. Seminars in cell \& developmental biology, 34, 133-139.

Ren, H., Zhuang, X., Cai, Y., Rabczuk, T., 2016. Dual-horizon peridynamics. Int. J. Numer. Meth. Engng 108, 1451-1476.

Silling, S.A., 2000. Reformulation of elasticity theory for discontinuities and long-range forces. Journal of the Mechanics and Physics of Solids 48, 175-209.

Silling, S.A., Epton, M., Weckner, O., Xu, J., Askari, E., 2007. Peridynamic states and constitutive modeling. Journal Elasticity 88, 151-184.
Stolarska, M.A., Kim, Y., Othmer, H.G., 2009. Multi-scale models of cell and tissue dynamics. Philosophical Transactions of the Royal Society A: Mathematical, Physical and Engineering Sciences $367,3525-3553$.

Su, Y., Chiang, P., Cheng, L., Lee, C., Swami, N. Chou, C., 2015. High aspect ratio nanoimprinted grooves of poly (lactic-co-glycolic acid) control the length and direction of retraction fibers during fibroblast cell division. Biointerphases 10, 041008.

Taber, L., 1995. Biomechanics of growth, remodeling, and morphogenesis. Applied mechanics reviews 48, $487-545$.

Tepole, A., 2016. Computational systems mechanobiology of wound healing. Computer Methods in Applied Mechanics and Engineering 10.1016/j.cma.2016.04.034.

Tepole, A.B., Kuhl, E., 2016. Computational modeling of chemo-bio-mechanical coupling: a systemsbiology approach toward wound healing. Computer methods in biomechanics and biomedical engineering 19, 13-30.

Théry, M., Jiménez-Dalmaroni, A., Racine, V., Bornens, M., Jülicher, F., 2007. Experimental and theoretical study of mitotic spindle orientation. $\mathrm{Na}$ ture $447,493-496$.

Williams, S., Ratliff, L., Postiglione, M.P., Knoblich, J., Fuchs, E., 2014. Par3-minsc and gai3 cooperate to promote oriented epidermal cell divisions through lgn. Nature cell biology 16, 758-69.

Wise, S., Lowengrub, J., Frieboes, H., Cristini, V., 2008. Three-dimensional multispecies nonlinear tumor growth-i: model and numerical method. Journal of theoretical biology 253, 524-543.

Wyatt, T., Harris, A., Lam, M., Cheng, Q., Bellis, J., Dimitracopoulos, A., Kabla, A., Charras, G., Baum, B., 2015. Emergence of homeostatic epithelial packing and stress dissipation through divisions oriented along the long cell axis. Proceedings of the National Academy of Sciences 112, 5726-5731. 
Zohdi, T., Holzapfel, G., Berger, S., 2004. A phenomenological model for atherosclerotic plaque growth and rupture. Journal of theoretical biology 227, 437-443.

Zöllner, A., Tepole, A., Kuhl, E., 2012. On the biomechanics and mechanobiology of growing skin. Journal of theoretical biology 297, 166-175. 